\title{
Improving Classification Accuracy in Through-Wall Radar Imaging Using Hybrid Prony's and Singular Value Decomposition Method
}

\author{
Anthony T. S. Ho, W. H. Tham and K. S. Low \\ School of Electrical and Electronic Engineering \\ Nanyang Technological University, \\ Nanyang Avenue, \\ Singapore 639798 \\ etsho@ntu.edu.sg
}

\begin{abstract}
This paper investigates the classification and identification of objects of different material behind a wall. The different materials are defined based on their permittivity, permeability and conductivity and include concrete, wood, glass and metal. A simulation program based on the finite difference time domain (FDTD) [1] method was first used to model the wall and objects behind the wall. An ultra-wideband (UWB) electromagnetic pulse was then transmitted through the wall towards the object. The receiver (on the same side of the wall as the transmitter) recorded the reflected signal which was subsequently analyzed using Prony's method [2].

Prony's method decomposes the transient signal into a sum of decaying sinusoids. These sinusoids are represented by their poles and residues. Detailed analysis of the poles and residues was then carried out with the aim of classifying them based on the material medium of the object.

It is known that the pole positions tend to become unstable and will shift when there is noise. Therefore, an investigation was also carried out on the effect of noise (with SNR from 20 to $40 \mathrm{~dB}$ ) on the pole positions. Singular value decomposition [3] was also used to enhance the pole stability in the presence of noise.
\end{abstract}

Keywords-Through-Wall Radar Imaging, Prony's method, Singular Value Decomposition, Classification, Identification.

\section{INTRODUCTION}

There are many occasions when it is desirable to inspect the interior of a room or building before physically entering it. This is especially so when entering the room might pose a serious danger to the person involved. These situations include civil engineering, search and rescue and law enforcement scenarios.

Through-wall radar imaging is the technology which addresses this problem and has become a keen research topic in recent years. Its importance is due to the increased emphasis on homeland security, which involves many key areas such as gathering intelligence, concealed weapons detection and domestic counter-terrorism. Other applications of through-wall radar imaging include urban warfare, hostage rescue scenarios as well as search and rescue operations.

Furthermore, the study and use of ultra-wideband (UWB) technology has also gained momentum in the last few years due to the approval of its use commercially by the Federal Communications Commission (FCC) in 2002. The large available bandwidth for UWB and its performance in dispersive environments make it ideal for indoor use in highclutter environments. Coupled with the fact that UWB provides high spatial resolution, the use of UWB in throughwall radar imaging can be seen as an obvious choice.

Analysis and classification of through-wall radar signals poses many difficulties, due to the presence of the wall as well as multiple reflections due to many objects within a room. It is thus desirable to extract certain dominant characteristics from the received signals which correspond to the different types of material within the room.

A computer program based on the finite difference time domain (FDTD) method was used to simulate the room environment as well as the transmitted UWB pulse. The received signal was then analyzed using Prony's method which decomposes the signal into its poles and residues. Pole positions and characteristics were analyzed to determine the "dominant" poles which correspond to certain material properties. Shifting of the poles, due to the addition of noise, was also studied. Singular value decomposition was used to improve on the pole stability.

Section II will introduce the use of FDTD to simulate the UWB pulse as well as the room environment.

Section III highlights Prony's method for signal decomposition.

Section IV will show the results obtained from Prony's decomposition of the received signals generated using the FDTD simulations.

\section{FINITE DIFFERENCE TIME DOMAIN (FDTD)}

\section{A. Maxwell's Equations}

Maxwell's equations for linear, isotropic, non-dispersive materials are 


$$
\begin{gathered}
\frac{\partial \mathbf{B}}{\partial t}=-\nabla \times \mathbf{E} \\
\frac{\partial \mathbf{D}}{\partial t}=\nabla \times \mathbf{H}-\mathbf{J}
\end{gathered}
$$

\section{B. Finite Difference Formulation}

Based on the notation and formulation in [4], taking only two dimensions (i.e. electric and magnetic fields do not vary in the z-direction) and considering only the transverse-electric mode $\left(\mathrm{TE}_{\mathrm{z}}\right)$, Maxwell's equations can be expressed as finite differences as

$$
\begin{aligned}
& \left.H_{z}\right|_{i+1 / 2, j+1 / 2} ^{n+1 / 2}=\left.H_{z}\right|_{i+1 / 2, j+1 / 2} ^{n-1 / 2}+ \\
& \frac{\Delta t}{\mu}\left[\begin{array}{l}
\frac{\left.E_{x}\right|_{i+1 / 2, j+1} ^{n}-\left.E_{x}\right|_{i+1 / 2, j} ^{n}}{\Delta y}- \\
\frac{\left.E_{y}\right|_{i+1, j+1 / 2} ^{n}-\left.E_{y}\right|_{i, j+1 / 2} ^{n}}{\Delta x}
\end{array}\right] \\
& \left.E_{x}\right|_{i+1 / 2, j} ^{n+1}=\left.\left(\frac{2 \varepsilon-\sigma \Delta t}{2 \varepsilon+\sigma \Delta t}\right) E_{x}\right|_{i+1 / 2, j} ^{n}+ \\
& \frac{2 \Delta t}{2 \varepsilon+\sigma \Delta t}\left[\frac{\left.H_{z}\right|_{i+1 / 2, j+1 / 2} ^{n+1 / 2}-\left.H_{z}\right|_{i+1 / 2, j-1 / 2} ^{n+1 / 2}}{\Delta y}\right] \\
& \left.E_{y}\right|_{i, j+1 / 2} ^{n+1}=\left.\left(\frac{2 \varepsilon-\sigma \Delta t}{2 \varepsilon+\sigma \Delta t}\right) E_{y}\right|_{i, j+1 / 2} ^{n}- \\
& \frac{2 \Delta t}{2 \varepsilon+\sigma \Delta t}\left[\frac{\left.H_{z}\right|_{i+1 / 2, j+1 / 2} ^{n+1 / 2}-\left.H_{z}\right|_{i-1 / 2, j+1 / 2} ^{n+1 / 2}}{\Delta x}\right]
\end{aligned}
$$

\section{Berenger's Perfectly Matched Layer (PML)}

A problem with using the FDTD method is that as the scattered electromagnetic fields reach the boundary of the simulation domain, they are reflected back, which results in undesirable interference. As a result, absorbing boundary conditions need to be applied at the boundary of the simulation domain to accurately simulate the electromagnetic waves propagating out into free space.

Absorbing boundary conditions developed by Berenger [5, 6] and Mur [7] have been used in many FDTD models to solve for the boundary problem. Due to its superior absorption of the electromagnetic waves at any frequency and incidence angle, Berenger's perfectly matched layer (PML) was chosen for the current simulation model.

\section{PRONY'S METHOD \& SINGULAR VALUE DECOMPOSITION (SVD)}

\section{A. Prony's Method}

Prony's method [8] decomposes a time signal into decaying exponential functions. By Prony's method, the sampled data $f(t)$ can be estimated as follows,

$$
f(t)=\sum_{v=1}^{N} C_{v} e^{S_{v} t}
$$

where $N$ is the number of poles and residues, $C_{v}$ are the residues and $S_{v}$ are the poles.

It can be shown that $f(t)$ satisfies the difference equation

$$
\sum_{p=0}^{N} a_{p} f_{p+k}=0, \quad k=0,1, \ldots, M-1
$$

Since $a_{N}=1$, (7) can be written as

$$
\left[\begin{array}{cccc}
f_{0} & f_{1} & \cdots & f_{N-1} \\
f_{1} & f_{2} & \cdots & f_{N} \\
\vdots & \vdots & & \vdots \\
f_{M-1-N} & f_{M-N} & \cdots & f_{M-1}
\end{array}\right]\left[\begin{array}{c}
a_{0} \\
a_{1} \\
\vdots \\
a_{N-1}
\end{array}\right]=-\left[\begin{array}{c}
f_{N} \\
f_{N+1} \\
\vdots \\
f_{M-1}
\end{array}\right]
$$

or more compactly as

$$
\mathbf{B a}=\mathbf{C}
$$

From (8), $a_{0}$ to $a_{N}$ will be known where $a_{p}$ are the coefficients of the algebraic equation, expressed as follows:

$$
\sum_{p=0}^{N} a_{p} z^{p}=0
$$

Solving the polynomial (10), the poles can thus be obtained by

$$
S_{v}=\frac{1}{\Delta t} \ln z_{v}
$$

Thus, $C_{v}$ can be found from (6)

\section{B. Singular Value Decomposition (SVD)}

Application of Prony's algorithm requires prior knowledge of the number of poles in the sampled data [9]. If lesser poles are used to estimate the data than the exact number, then the resulting poles will probably be inaccurate. If more poles are used than are contained in the sampled data, then the extra poles will cause the residues of the true poles to be inaccurate 
and also result in excess in computation time.

Furthermore, when noise is added, the matrix $\mathbf{B}$ in (9) will take on its full rank regardless of the actual number of poles it contains. This results in the inaccurate estimation of the true poles.

SVD [10] can be used to find the best lower rank approximation to the matrix $\mathbf{B}$, thus reducing the effects of noise on the shift in the pole positions.

Using SVD, the matrix $\mathbf{B}$ can be decomposed as

$$
\mathbf{B}=\mathbf{U} \boldsymbol{\Sigma} \mathbf{V}^{*}
$$

where $\boldsymbol{\Sigma}$ is a diagonal matrix with non-negative values and $\mathbf{U}$ and $\mathbf{V}^{*}$ are unitary matrices.

The diagonal entries of $\boldsymbol{\Sigma}$ are called the singular values of B . In the noiseless case, there will be $N$ number of nonnegative values corresponding to the $N$ number of true poles and the rest of the singular values will be zero. With the addition of noise, the previously zero values will now have small non-zero values, thus increasing the rank of $\mathbf{B}$.

Normally, the small non-zero singular values caused by the noise are generally a few magnitudes smaller than the other $N$ singular values due to the actual poles. Thus, to correct for the addition of noise, simply replace $\mathbf{B}$ with

$$
\hat{\mathbf{B}}=\mathbf{U} \hat{\boldsymbol{\Sigma}} \mathbf{V}^{*}
$$

where $\hat{\boldsymbol{\Sigma}}$ is obtained from $\boldsymbol{\Sigma}$ by setting to zero all singular values which are several magnitudes below the largest value.

\section{IV.RESULTS}

\section{A. Setup Parameters}

Figure 1 shows the transmitter, receiver, wall and object positions in the simulation domain. A gypsum wall $\left(\varepsilon_{\mathrm{r}}=2.8\right.$, $\sigma=0.15$ ) was used.

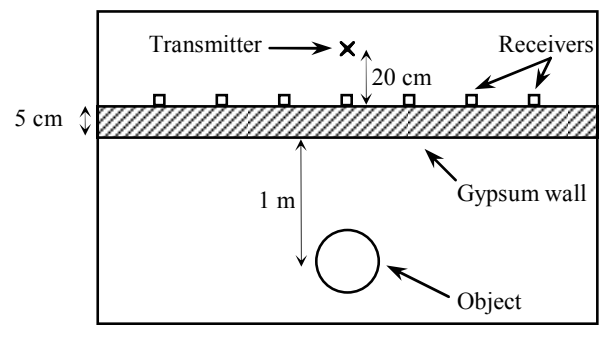

Figure 1 Layout of the simulation domain

\section{B. UWB Input Pulse}

The transmitted UWB pulse is given by

$$
x(t)=\cos \left(2 \pi f_{c} t\right) \exp \left(-\frac{t^{2}}{u^{2}}\right)
$$

where $f_{c}$ is the center frequency and $u$ determines the bandwidth of the pulse. For our simulation, a pulse with center frequency $4 \mathrm{GHz}$ and bandwidth of $2 \mathrm{GHz}$ was used.

\section{Received Signal}

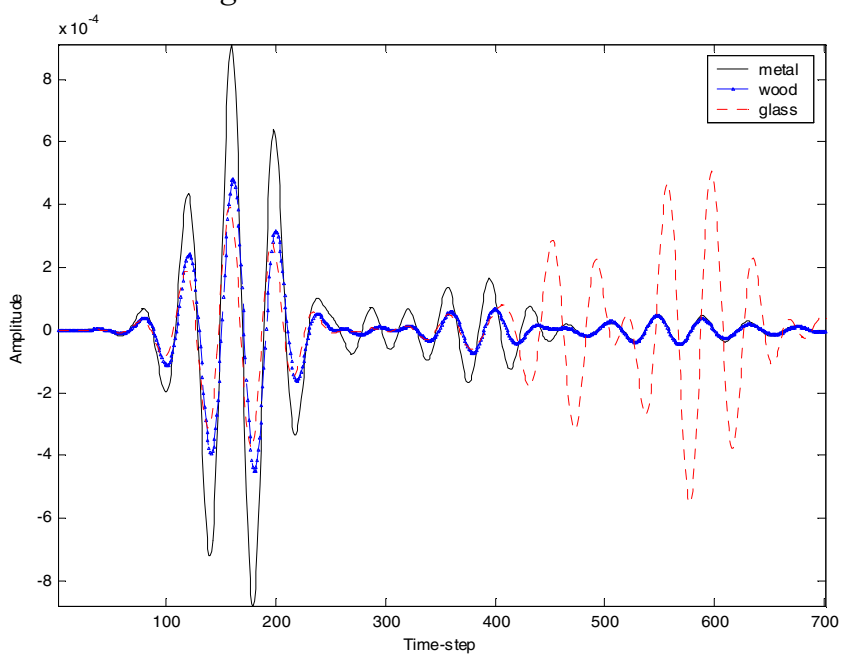

Figure 2. Received signal for different object materials

The UWB pulse was transmitted through the wall and the receivers recorded its reflected signal from the object. Three different materials were used for the object, namely metal, wood and glass. The received signal at the center receiver for different object materials is shown in Fig. 2. Note that the signal has been time-gated to show only the portion where the transmitted pulse interacted with the object.

As can be seen in Fig. 2, the received signal for the metal object has the highest amplitude due to the high reflectivity of metal. As such, the received signal for the metal object will be used to demonstrate signal decomposition using Prony's method in the next section.

\section{Effect of Noise on the Pole Positions}

To obtain the pole positions, Prony's method was applied to the received signal for the metal object. As discussed in Section III, the pole positions are dependent on the initial estimate of the number of poles used. Also, the addition of noise will cause a further shift in the pole positions.

The pole positions of the received signal with signal-tonoise ratio (SNR) of $40 \mathrm{~dB}$ was obtained using 280, 290 and 300 initial number of poles (Fig. 3(a)). Note that only the first 20 poles with the highest magnitude were plotted.

Due to the high SNR, the pole positions remained the same regardless of the initial pole estimates. With a SNR of $30 \mathrm{~dB}$ (Fig. 3(b)), there is a small shift in a few of the poles. Finally, with a noisy signal at $20 \mathrm{~dB}$, several poles can be seen to have shifted (Fig. 3(c)).

\section{E. Pole Stability}

Stable or "dominant" poles are the poles which do not shift even at low SNR. These are the poles which are needed to classify and identify different received signals from different objects. Ideally, most of the poles should be stable to form a good classification. However, in the presence of noise, this may not be so. 
Fig. 4 shows the pole positions for the received signal with different SNR using an initial estimate of 300 poles. Note that Fig. 4 is an enlargement of the second-quadrant where the poles are clustered near the center. As can be seen, there are several poles which do not shift and are thus considered stable.
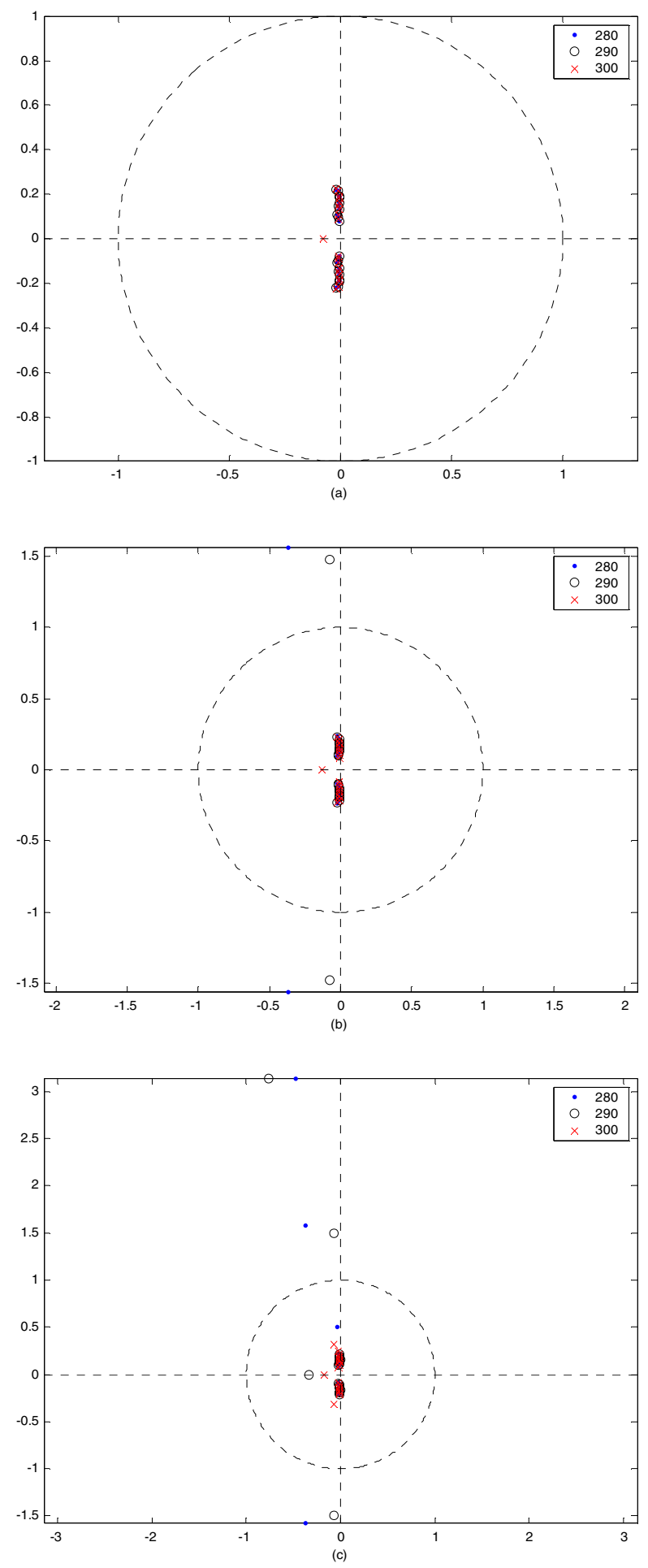

Figure 3. Pole positions for received signal with SNR of (a) $40 \mathrm{~dB}$ (b), $30 \mathrm{~dB}$ and (c) $20 \mathrm{~dB}$.

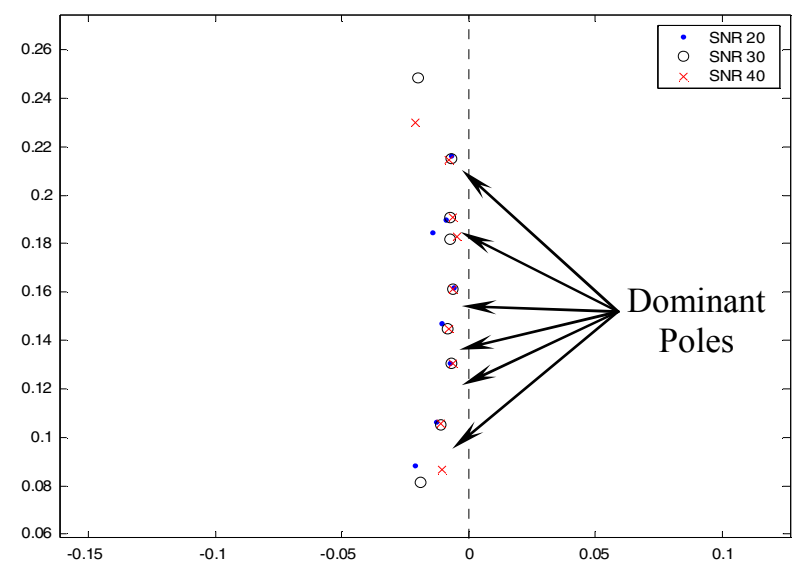

Figure 4. "Dominant" poles of the received signal

\section{CONCLUSION}

A simulation has been done to model the transmission of a UWB pulse for the purpose of through-wall imaging. Classification of the received signal was carried out by studying the pole patterns obtained using Prony's method.

A study was also carried out on the shift in pole positions in the presence of noise. Results show that at noise levels of 20 , 30 and $40 \mathrm{~dB}$, there were still a number of poles dominant that remain stable at their respective locations. Future work will involve improvements in the pole stability even for signals with low SNRs.

\section{REFERENCES}

[1] K. S. Yee, "Numerical solutions of initial boundary value problems involving Maxwell's equations in isotropic media," IEEE Trans. Antennas Propagat., vol. AP-14, pp. 310-317, May 1966.

[2] A. J. Poggio, M. L. Van Blaricum, E. K. Miller and R. Mittra, "Evaluation of a processing technique for transient data," IEEE Trans. Antennas Propagat., vol. AP-26, pp. 165-173, Jan 1978.

[3] R. Kumaresan and D. W. Tufts, "Estimating the parameters of exponentially damped sinusoids and pole-zero modeling in noise," IEEE Trans. Acoustics, Speech and Signal Processing, vol. ASSP-30, pp. 833840, Dec. 1982.

[4] A. Taflove and Susan C. Hagness, Computational Electrodynamics: The Finite-Difference Time-Domain Method, $2^{\text {nd }}$ Edition, Artech House, 2000.

[5] J. P. Berenger, "A Perfectly Matched Layer for the Absorption of Electromagnetic Waves," Journal of Computational Physics, vol. 114 pp. 185-200, 1994.

[6] J. P. Berenger, "Perfectly matched layer for the FDTD solution of wavestructure interaction problems," IEEE Trans. Antennas Propagat., vol. 44, pp. 110-117, Jan 1996.

[7] G. Mur, "Absorbing boundary conditions for finite-difference approximation of the time-domain electromagnetic-field equations," IEEE Trans. Electromagn. Compat., vol. 23, pp. 1073-1077, 1981.

[8] A. J. Poggio, M. L. Van Blaricum, E. K. Miller and R. Mittra, "Evaluation of a Processing Technique for Transient Data", IEEE Trans. Antennas and Propagat., vol. 26, issue 1, pp. 165 - 173, 1978.

[9] M. L. Van Blaricum and R. Mittra, "Problems and Solutions Associated with Prony's Method for Processing Transient Data", IEEE Trans. Antennas and Propagat., vol. 26, issue 1, pp. $174-182,1978$.

[10] D. W. Tufts and R. Kumaresan, "Singular Value Decomposition and Improved Frequency Estimation Using Linear Prediction", IEEE Trans. Acoustics, Speech and Signal Proc., vol 30, issue 4, pp. 671 - 675, 1982. 